\title{
PossDL - A Possibilistic DL Reasoner for Uncertainty Reasoning and Inconsistency Handling
}

\author{
Guilin $\mathrm{Qi}^{1}$, Qiu Ji ${ }^{1}$, Jeff Z. Pan ${ }^{2}$, and Jianfeng $\mathrm{Du}^{3}$ \\ 1 School of Computer Science and Engineering, Southeast University, Nanjing 210096 \\ ${ }^{2}$ Institute AIFB, University of Karlsruhe, Karlsruhe, Germany \\ 3 Department of Computing Science, University of Aberdeen, Aberdeen, UK \\ ${ }^{4}$ Guangdong University of Foreign Studies, Guangzhou 510006, China
}

\begin{abstract}
Uncertainty reasoning and inconsistency handling are two important problems that often occur in the applications of the Semantic Web. Possibilistic description logics provide a flexible framework for representing and reasoning with ontologies where uncertain and/or inconsistent information exists. Based on our previous work, we develop a possibilistic description logic reasoner. Our demo will illustrate functionalities of our reasoner for various reasoning tasks that possibilistic description logics can provide.
\end{abstract}

\section{Introduction}

Uncertainty reasoning and inconsistency handling are two important problems that often occur in the applications of the Semantic Web, such as the areas like medicine and biology [4]. Recently, there is an increasing interest to extend Web Ontology Language OWL to represent uncertain knowledge. Most of the work is based on Description Logics (DL) that provide important formalisms for representing and reasoning with ontologies. A DL knowledge base is then extended by attaching each axiom in it with a degree of belief. The degree of belief can have several meanings depending on the semantics of the logic. For example, in probabilistic description logics, the degree of belief can be explained as degree of overlap between two concepts and in possibilistic description logics [4], the degree of belief is explained as the necessity degree or certainty degree (see [7]). Inconsistency handling in DL is another problem that has attracted a lot of attention. Inconsistency can occur due to several reasons, such as modeling errors, migration or merging ontologies, and ontology evolution. When an ontology is inconsistent, an ontology language which has first-order features, such as a description logic, cannot be applied to infer non-trivial conclusions.

Let us consider a medical ontology modified from an example given in [4].

Example 1. Given an ontology $\mathcal{B}$ consisting of the following terminological axioms attached with confidence degrees:

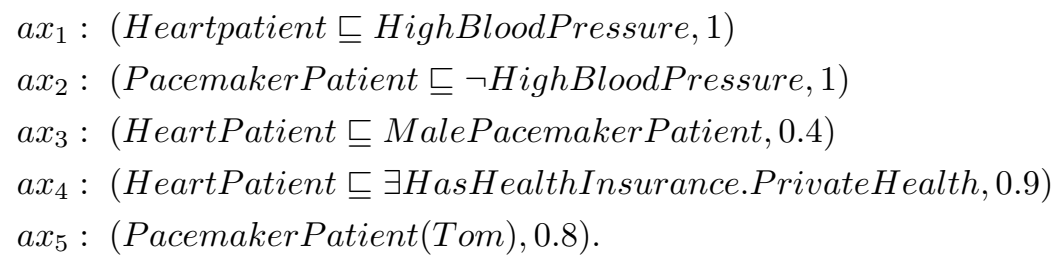


Suppose we use possibilistic logic, then $a x_{1}$ means that "it is absolute certain that heart patients suffers from high blood pressure", $a x_{2}$ can be explained similarly, $a x_{3}$ says that "it is a little certain that heart patient are male pacemaker patient", $a x_{4}$ says "it is highly certain that heart patients have a private insurance", and finally $a x_{5}$ states that "it is quite certain that Tom is a pacemaker patient". Suppose we learn that Tom is a heart patient with degree 0.5 ( $a x_{6}$ : (HeartPatient(Tom),0.5)), i.e., it is somewhat certain that Tom is a heart patient, and we add this axiom to the ontology, then the ontology will become inconsistent. From this updated ontology, we may want to query if Tom suffers from high blood pressure and to ask to what degree we can infer this conclusion?

Possibilistic description logics, first proposed by Hollunder in [2] and further developed by Qi and Pan in [7], are extensions of description logics with possibilistic semantics. It is well-known that possibilistic logic is a powerful logical framework for dealing with uncertainty and handling inconsistency. Possibilistic description logics inherit these two nice properties and have very promising applications in the Semantic Web. A possibilistic DL knowledge base consists of a set of weighted axioms of the form $(\phi, \alpha)$, where $\phi$ is a DL axiom such as an assertional axiom of the form $C(a)$ and $\alpha$ is an element of the semi-open real interval $(0,1]$ or of a finite total ordered scale. A weighted axiom $(\phi, \alpha)$ encodes the constraint $N(\phi) \geq \alpha$, where $N$ is a necessity measure [1], with the intended meaning that the necessity degree of $\phi$ is at least $\alpha$.

In our previous work [7], we have provided syntax and semantics of possibilistic description logics and defined several inference services. We have also provided algorithms for implementing these inference services. Based on these algorithms, in this work, we develop a possibilistic description logic reasoner by using OWL API 3.0.0.v1310 and Pellet v2.0.01. Our demo will illustrate functionalities of our reasoner for various reasoning tasks that possibilistic description logics can provide.

\section{The PossDL Reasoner}

\subsection{Possibilistic Description Logics}

We introduce the syntax of possibilistic DLs and some reasoning tasks, and refer to [7] for the semantics of possibilistic DLs. A possibilistic axiom is a pair $(\phi, \alpha)$ consisting of an axiom $\phi$ and a weight $\alpha \in(0,1]$ denoting the confidence degree of $\phi$, which will be interpreted as the necessity degree of $\phi$. A possibilistic TBox (resp., ABox) is a finite set of possibilistic axioms $(\phi, \alpha)$, where $\phi$ is an TBox (resp., ABox) axoim. A possibilistic DL knowledge base $\mathcal{B}=(\mathcal{T}, \mathcal{A})$ consists of a possibilistic TBox $\mathcal{T}$ and a possibilistic $\mathrm{ABox} \mathcal{A}$. Let $\mathcal{B}_{\alpha}=\left\{\phi_{i} \mid\left(\phi_{i}, \alpha_{i}\right) \in \mathcal{B}, \alpha_{i} \geq \alpha\right\}$. An important reasoning task in possibilistic DLs is to compute the inconsistency degree of a possibilistic DL knowledge base $\mathcal{B}$, denoted by $\operatorname{Inc}(\mathcal{B})$, which is defined as $\operatorname{Inc}(\mathcal{B})=\max \left\{\alpha_{i} \mid \mathcal{B}_{\geq}\right.$is inconsistent $\}$. Consider Example 1, suppose $\mathcal{B}=\left\{a x_{1}, \ldots, a x_{6}\right\}$, then $\operatorname{Inc}(\mathcal{B})=0.5$.

There are three inference services in possibilistic DLs.

- A DL axiom $\phi$ is a plausible consequence of a possibilistic DL knowledge base $\mathcal{B}$, written $\mathcal{B}=_{P} \phi$ if $\mathcal{B}_{>\operatorname{Inc}(\mathcal{B})} \models \phi$.

${ }^{1}$ http://clarkparsia.com/pellet/ 
- A DL axiom $\phi$ is a possibilistic consequence of $\mathcal{B}$ to degree $\alpha$, written $\mathcal{B} \models_{\pi} \phi$, if the following conditions hold: (1) $\mathcal{B}_{\geq \alpha}$ is consistent, (2) $\mathcal{B}_{\geq \alpha} \models \phi$, (3) for all $\beta>\alpha$, $\mathcal{B}_{\geq \beta} \not \neq \phi$.

- A possibilistic DL axiom $(\phi, \alpha)$ is a consequence from $\mathcal{B}$, written $\mathcal{B}=(C(a), \alpha)$, if $\alpha>\operatorname{Inc}(\mathcal{B})$ and $\mathcal{B}_{\geq \alpha} \models \phi$.

Note that the possibilistic consequence relation $\models_{\pi}$ is different from the consequence relation $=$ because the former is to check to what degree an assertion holds whilst the latter is to check if a possibilistic assertion holds. Consider Example 1 again, we have $\mathcal{B} \models_{P} \neg$ HighBloodPressure $($ Tom $), \mathcal{B} \models_{\pi}(\neg$ HighBloodPressure $($ Tom $), 0.8)$, and $\mathcal{B}=($ Heartpatient $\sqsubseteq$ HighBloodPressure, 1$)$. However, HeartPatient $\sqsubseteq$ MalePacemakerPatient cannot be inferred from $\mathcal{B}$ by possibilistic inference due to the notorious drowning effect, i.e., all axioms whose degrees are less than or equal to $\operatorname{Inc}(\mathcal{B})$ are blocked to be used in the inference. Therefore, we also implement a drowning-free variant of possibilistic inference, called linear order inference. Let $\mathcal{B}=$ $\left\{\left(\phi_{i}, \alpha_{i}\right): i=1, \ldots, n\right\}$ be a possibilistic DL knowledge base. Suppose $\beta_{j}(j=1, \ldots, k)$ are all distinct necessity degrees appearing in $\mathcal{B}$ such that $\beta_{1}>\beta_{2}>\ldots>\beta_{k}$. Let $\Sigma_{\mathcal{B}}=\left(S_{1}, \ldots, S_{k}\right)$, where $S_{i}=\left\{\phi_{l}:\left(\phi_{l}, \alpha_{l}\right) \in \mathcal{B}, \alpha_{l}=\beta_{i}\right\}$, and $\Sigma_{L O, \mathcal{B}}=\bigcup_{i=1}^{k} S_{i}^{\prime}$, where $S_{i}^{\prime}$ is defined by $S_{i}^{\prime}=S_{i}$ if $S_{i} \cup \bigcup_{j=1}^{i-1} S_{j}^{\prime}$ is consistent, $\emptyset$ otherwise. A DL axiom $\phi$ is said to be a linear consequence of $\mathcal{B}$, denoted by $\left.\mathcal{B}\right|_{L O} \phi$, if and only if $\Sigma_{L O, \mathcal{B}} \models \phi$. In Example 1, we have $\mathcal{B}={ }_{L O}$ HeartPatient $\sqsubseteq$ MalePacemakerPatient.

\subsection{The PossDL Reasoner}

We have developed a tool, called PossDL, as a plug-in in NeOn Toolkit v2.3 $3^{2}$ which is a multi-platform ontology engineering environment. The PossDL reasoner provides the functionalities of computing inconsistency degree and doing instance / subsumption checking with necessity degree for a possibilistic DL knowledge base. It takes an OWL ontology and a separate file storing the necessity degrees for the axioms in the ontology as inputs. PossDL mainly consists of four parts, described as follows.

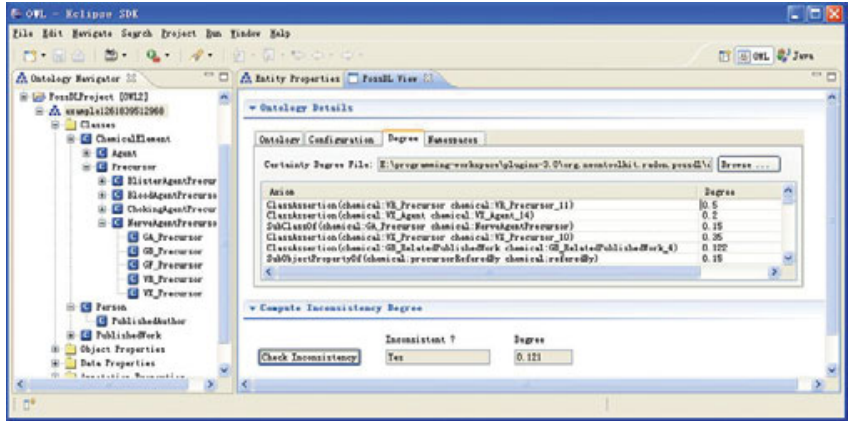

Fig. 1. The user interface to compute inconsistency degree

${ }^{2}$ http://neon-toolkit.org/wiki/Main_Page 


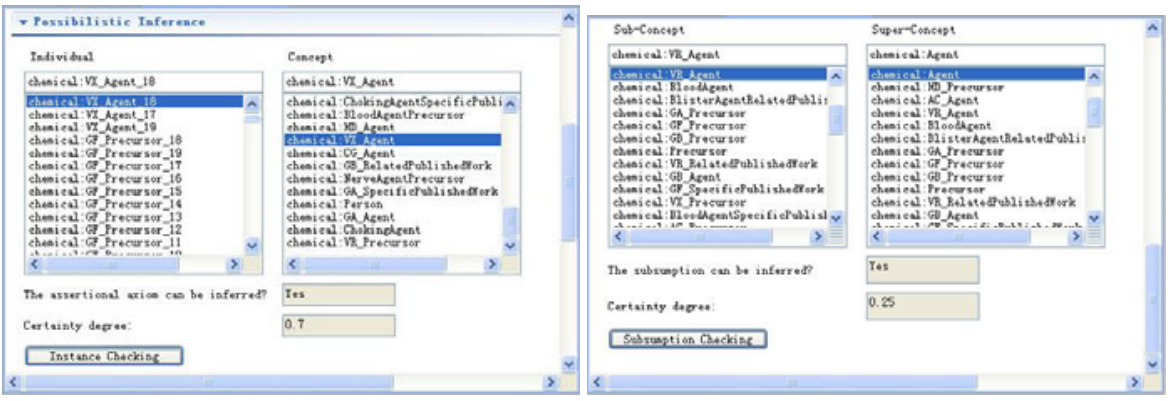

Fig. 2. The user interfaces for instance / subsumption checking using possibilistic inference

In the first part (see "Ontology Details" in Figure1), we show the information about the chosen ontology $O$ (see "Ontology" tab), the necessity degrees for the axioms in $O$ (see "Degree" tab), the namespaces appearing in $O$ (see "Namespaces" tab) and the configuration (see "Configuration" tab). In the "Configuration" tab, we provide three options about the reasoning tasks: compute inconsistency degree, possibilistic inference and linear order inference. If one of them is chosen, the corresponding part or section will be shown automatically. By default, the first option (i.e. compute inconsistency degree) is chosen. It is noted that, the necessity degrees are stored in a separate file. This file is still in OWL format. Specifically, we take a string of an axiom as a concept ID and associate a necessity degree to this concept by an annotation property.

In the part of "Compute Inconsistency Degree" (see "Compute Inconsistency Degree" in Figure 1, we can compute the inconsistency degree by clicking the button. If this possiblisitic DL knowledge base is inconsistent, "yes" will be shown in the text area below the sentence of "Inconsistency?" and the inconsistency degree can be seen in the text area right below the word of "Degree". Otherwise, "no" and "-" will be shown in two text areas respectively.

In the part of "Possibilistic Inference" (see the left part in Figure2), we can choose an individual and a concept to do instance checking with necessity degree based on the possibilistic inference by clicking the button of "Instance Checking". The result about whether the corresponding assertional axiom can be inferred will be shown in the first text area. If this axiom can be inferred, the necessity degree for this axiom will be computed and it can be seen in the second text area. Otherwise, "-" will be shown. It is similar to do subsumption checking in this part (see the right part in Figure 2). The part of "Linear Order Inference" is similar to the part of "Possibilistic Inference" but using different inference strategies.

\section{Related Work}

The relationship between possibilistic DLs and other uncertainty formalisms for DLs has been discussed in a survey paper [5]. One of the most important approaches that extend DLs with uncertainty reasoning are probabilistic DLs, such as the work presented in [4] which has a tool support [3]. Some major differences between possibilistic DLs and probabilistic DLs are given as follows. First, unlike probabilistic DLs, the confidence degree attached to an axiom in possibilistic DLs is not absolute and can be replaced by another 
number as long as the ordering between two confidence degrees is not changed. Second, in possibilistic DLs, a necessity degree is attached to a DL axiom. Whist in probabilistic DLs in [4], an interval $[l, u](l, u \in[0,1])$ is attached to a conditional constraint $(D \mid C)$, where $C$ and $D$ are DL concepts, which cannot be expressed by means of DL axioms.

Fuzzy DLs can be used to deal with uncertainty or vagueness in DLs (e.g., [9.8]) with scalable tool support [6]. The main difference between possibilistic DLs and fuzzy DLs is that, the truth value of a concept (or a role) in possibilistic DLs is still twovalued, whilst in fuzzy DLs, the truth value of a concept (or a role) is multi-valued. So the semantics of possibilistic DLs is different from that of fuzzy DLs.

\section{What Will Be Demonstrated?}

In our demonstration, we present our PossDL reasoner, which is an extension of Pellet for uncertainty reasoning and inconsistency handling. In particular, PossDL supports three different possibibilistic inference services and the linear order inference which is used to deal with the drowning effect. In the demonstration, we will illustrate our reasoner with practical examples obtained from ontology learning and ontology matching. Besides, for various needs of the users different algorithms to deal with inconsistency and/or uncertainty will be demonstrated.

\section{Acknowledgments}

Guilin Qi is partially supported by Excellent Youth Scholars Program of Southeast University under grant 4009001011.

\section{References}

1. Dubois, D., Lang, J., Prade, H.: Possibilistic logic. In: Handbook of Logic in Aritificial Intelligence and Logic Programming, pp. 439-513 (1994)

2. Hollunder, B.: An alternative proof method for possibilistic logic and its application to terminological logics. In: Proc. of UAI 1994, pp. 327-335 (1994)

3. Klinov, P., Parsia, B.: Optimization and evaluation of reasoning in probabilistic description logic: Towards a systematic approach. In: Sheth, A.P., Staab, S., Dean, M., Paolucci, M., Maynard, D., Finin, T., Thirunarayan, K. (eds.) ISWC 2008. LNCS, vol. 5318, pp. 213-228. Springer, Heidelberg (2008)

4. Lukasiewicz, T.: Expressive probabilistic description logics. Artif. Intell. 172(6-7), 852-883 (2008)

5. Lukasiewicz, T., Straccia, U.: Managing uncertainty and vagueness in description logics for the semantic web. Journal of Web Semantics 6(4), 291-308 (2008)

6. Pan, J.Z., Stamou, G.B., Stoilos, G., Taylor, S., Thomas, E.: Scalable querying services over fuzzy ontologies. In: Proc. of WWW 2008, pp. 575-584 (2008)

7. Qi, G., Pan, J.Z., Ji, Q.: Extending description logics with uncertainty reasoning in possibilistic logic. In: Mellouli, K. (ed.) ECSQARU 2007. LNCS (LNAI), vol. 4724, pp. 828-839. Springer, Heidelberg (2007)

8. Stoilos, G., Stamou, G., Pan, J.Z., Tzouvaras, V., Horrocks, I.: Reasoning with very expressive fuzzy description logics. J. Artif. Intell. Res. 30, 273-320 (2007)

9. Straccia, U.: Reasoning within fuzzy description logics. J. Artif. Intell. Res. 14, 137-166 (2001) 